Musées, Patrimoine et Culture scientifiques et techniques

$174 \mid 2017$

novembre-décembre 2017

\title{
Recensement, conservation et valorisation du patrimoine professionnel de la Ville de Paris
}

\author{
Miriam Simon
}

\section{OpenEdition}

\section{Journals}

Édition électronique

URL : https://journals.openedition.org/ocim/4285

DOI : $10.4000 /$ ocim.4285

ISSN : 2108-646X

Éditeur

OCIM

Édition imprimée

Date de publication : 1 novembre 2017

Pagination : 25-31

ISSN : 0994-1908

Référence électronique

Miriam Simon, «Recensement, conservation et valorisation du patrimoine professionnel de la Ville de Paris », La Lettre de I'OCIM [En ligne], 174 | 2017, mis en ligne le 01 novembre 2018, consulté le 12 mai 2021. URL : http://journals.openedition.org/ocim/4285 ; DOI : https://doi.org/10.4000/ocim.4285

Ce document a été généré automatiquement le 12 mai 2021.

Tous droits réservés 


\title{
Recensement, conservation et valorisation du patrimoine professionnel de la Ville de Paris
}

\author{
Miriam Simon
}

Maquette de présentation des projets pour la passerelle Simone de Beauvoir (2000), DVD/CMA Centre de maintenance et d'approvisionnement

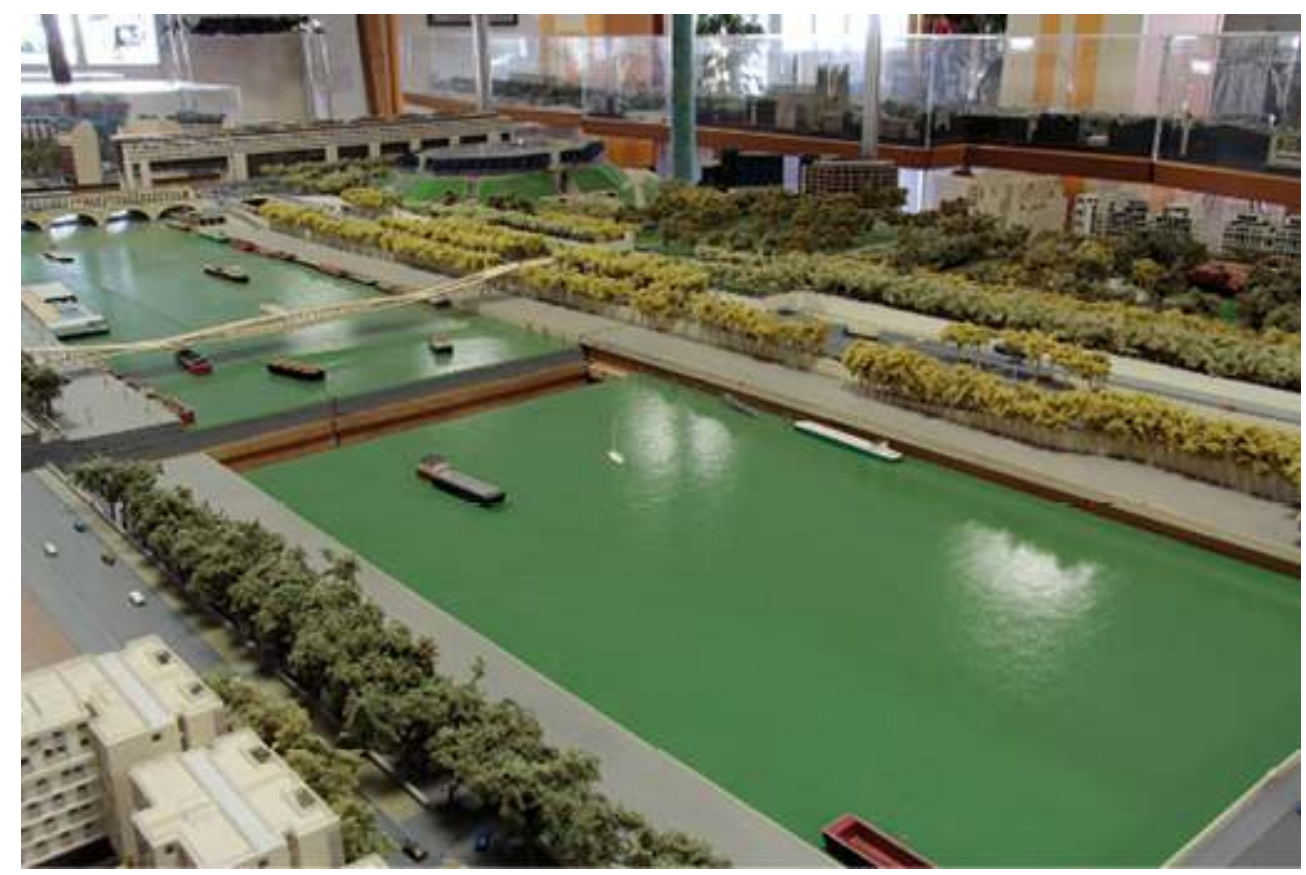

(c) Mairie de Paris/M. Simon

2 Soutenue par la Maire de Paris, la décision de créer une mission transversale de recensement, conservation et valorisation du patrimoine professionnel municipal s'inscrit dans un processus initié en 2015 par le Maire adjoint chargé des ressources humaines, des services publics et de la modernisation de l'administration, le Secrétaire 
Général de la Ville de Paris, et porté par le Directeur Général de la Propreté et de l'Eau (DPE). Les lettres de mission résument ainsi le diagnostic et la demande de conservation et de valorisation du patrimoine professionnel, englobant les dimensions matérielles et immatérielles de ce patrimoine centré sur les métiers qui s'exercent au sein de la fonction publique parisienne : "forte de ses missions de service public, la Ville de Paris dispose en effet d'un patrimoine matériel et immatériel relatif à leur exercice, d'une richesse et d'une variété exceptionnelles. En l'absence d'une politique de conservation définie, le patrimoine matériel fait jusqu'à présent l'objet d'un stockage empirique ou bien il est jeté en raison de son obsolescence. En outre, l'accélération des évolutions technologiques et les mutations de l'organisation administrative entament le sentiment de cohésion des équipes, le sens que donnent les agents à leur activité professionnelle, et la mémoire des métiers. Ce patrimoine pourrait faire l'objet d'une valorisation aux multiples dimensions, tant d'ordre historique, identitaire, que touristique et culturel. Dans cette perspective, il convient d'en prendre connaissance de manière précise, en recourant aux compétences d'un conservateur du patrimoine". La décision de professionnaliser la démarche entend donner à celle-ci une valeur ajoutée scientifique et technique. Ainsi l'ajout du volet de recensement à celui, initialement envisagé, de conservation et de valorisation, répond-il à l'acquiescement que pour conserver et valoriser, il faut connaître.

3 Sans recul suffisant pour procéder à une analyse permettant de théoriser l'expérience, nous nous bornerons dans cet article à une présentation chronologique.

\section{Lancement de la mission}

4 La mission a débuté en octobre 2015, avec le recrutement en interne d'un conservateur du patrimoine doté d'une expérience au sein de l'administration parisienne et dans la collecte, la conservation et la valorisation de patrimoine artistique et technique. Face à un périmètre d'une telle envergure, il s'agissait dans un premier temps de prendre la mesure d'un tel projet et de tenter des définitions, à commencer par celle du patrimoine professionnel. Il s'agit de l'ensemble des biens matériels et immatériels permettant l'exercice d'une activité professionnelle, témoins de la culture métier, du travail et des relations sociales dans le passé et le présent ${ }^{1}$. Ce champ se répartit en une multitude de sites (on en dénombre plusieurs centaines), situés dans Paris intra-muros et dans la banlieue. Il concerne une immense typologie d'équipements et d'objets (objets utilisés et objets fabriqués ou adaptés par des agents municipaux). Il se situe à la croisée de domaines variés puisqu'il concerne à la fois ces objets, des archives diverses, des expériences et des savoir-faire centrés sur l'exercice de métiers, mais aussi un patrimoine bâti, urbain (décors, équipements, infrastructures), paysager. Aussi est-il nécessaire de l'aborder de manière pluridisciplinaire, en convoquant l'histoire administrative, technique, scientifique et industrielle, la sociologie, l'ethnologie et l'anthropologie, la géographie et l'urbanisme, tout en requérant des compétences dans le domaine de la conservation matérielle.

5 Cette prise de mesure a été facilitée par l'immersion dans une direction technique l'une des plus grosses en termes d'effectifs (7 600 agents), très hiérarchisée, composée d'une forte population ouvrière et technique - la Direction de la Propreté et de l'Eau. Elle a bénéficié du soutien et de la confiance de son Directeur Général, qui a mis à la disposition de la mission des moyens logistiques, un budget de formation, du matériel photographique, vidéo et informatique. Nombre de contacts ont été pris au sein de la 
Ville, avec le service des archives notamment, afin de délimiter les périmètres respectifs (la mission se concentrant sur le repérage et la collecte d'objets en 3D), et d'anticiper la pérennisation des données numériques à venir. Dans cette attente, nous procédons à la conception d'une Gestion électronique des données (GED) avec l'appui de la Direction des systèmes et technologies de l'information (DSTI), et stockons les données créées sur des disques durs externes et sur les serveurs de la Ville de Paris.

Urnes de l'emprunt municipal (XXe siècle), DFA/Cité administrative

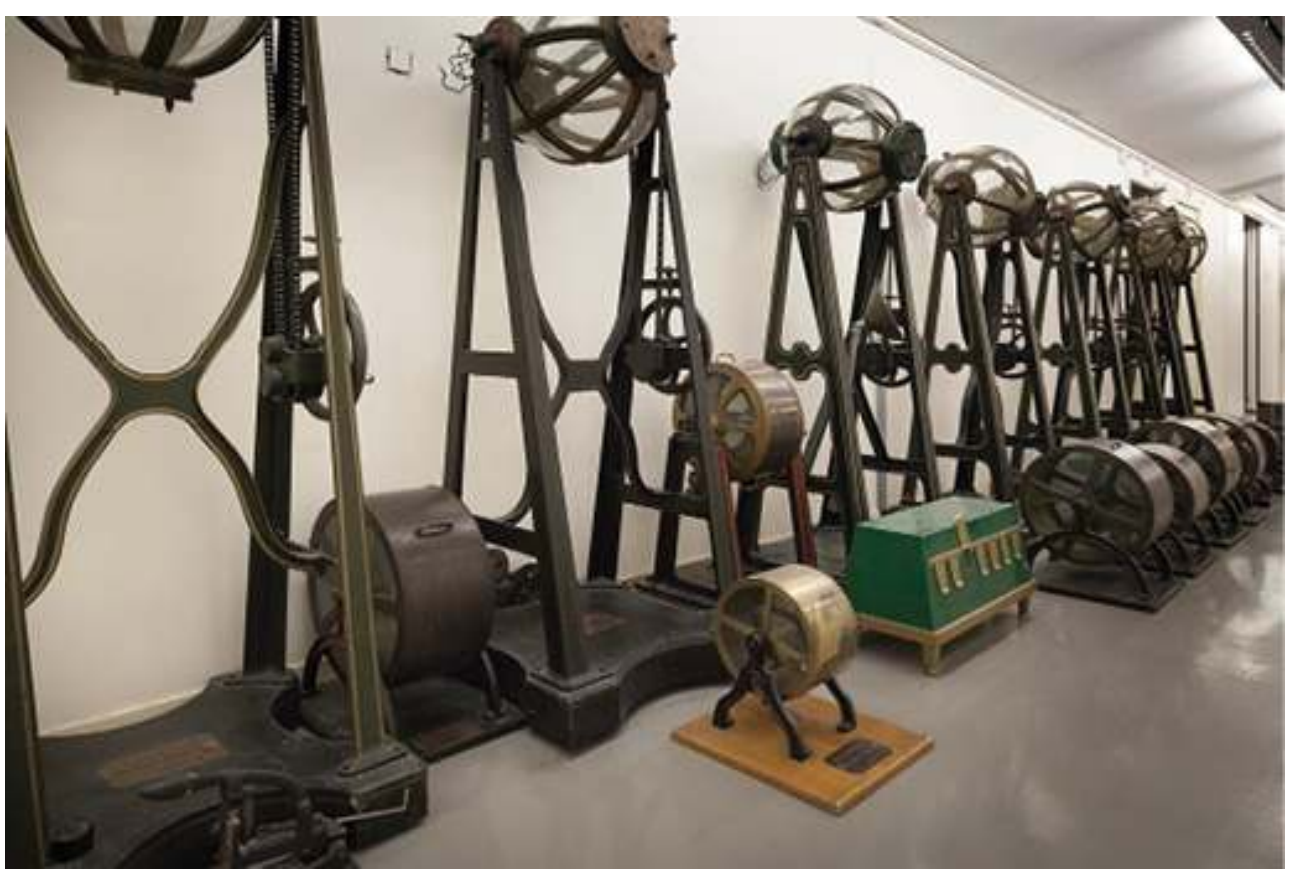

(C) Mairie de Paris/F. Morisson

De nombreux sites municipaux ont été visités, parmi les plus emblématiques et susceptibles de receler un patrimoine professionnel important, où l'accueil a généralement été à la mesure d'un fort désir de transmission. Parallèlement, des contacts ont été pris avec d'autres organisations de taille comparable, les milieux du patrimoine technique, scientifique et industriel, le monde universitaire et de la recherche en sciences sociales, afin de prendre connaissance des actions menées ailleurs en la matière, de bénéficier d'un appui méthodologique et dans une visée de recherche de partenariats. Dans le même temps, il s'agissait d'acquérir des connaissances par le moyen de recherches bibliographiques, tout en procédant à des mises au point juridiques (droits d'auteur) et informatiques (prospection de bases de données et de systèmes de gestion informatiques internes et externes).

\section{Ingénierie patrimoniale}

7 La mise en œuvre de tels objectifs supposait de mener au préalable une mission d'ingénierie patrimoniale. Pour ce faire, nous avons transposé la définition de l'ingénierie culturelle selon Claude Mollard : la "capacité d'apporter des solutions optimales, en termes de qualité, de coûts et de délais, aux demandes exprimées par les partenaires de la vie culturelle pour la définition d'objectifs, la mise en cuvre de programmes, la mobilisation de financements et la réalisation technique de projets". Ce temps de l'étude 
préalable est stratégique : il permet de repérer les acteurs, l'environnement social, de voir ce qui se pratique ailleurs, de cerner la demande, de faire l'effort conceptuel de définir ce que l'on veut savoir, ce que l'on cherche, à qui l'on s'adresse, de définir des corpus et des méthodologies.

C'est pourquoi il a été décidé de rédiger un rapport, adressé en mars 2017 par le Directeur Général de la DPE au Maire adjoint chargé des ressources humaines, des services publics et de la modernisation de l'administration, et au Secrétaire Général de la Ville de Paris. Ce document présente une analyse du contexte et de la commande, suivie de préconisations, puis de la description des actions menées jusqu'aux arbitrages attendus. De ces analyses et préconisations découlent des propositions de scenarii cohérentes et échelonnées.

L'histoire de Paris, capitale d'un pays très centralisé, tend à se confondre avec celle du pays, en dépit d'une émancipation récente et toujours en cours vis-à-vis de l'État. De cette tradition de centralisation découlent des tensions entre un souci de rayonnement national et international, et la gestion d'un quotidien de ville dont les évolutions récentes en faveur de la déconcentration et de la décentralisation veulent aller au plus près à la rencontre des préoccupations locales des Parisiens. On retrouve cette tradition centralisée dans l'organisation administrative municipale et le fonctionnement des directions, techniques en particulier, très pyramidal, usant d'une terminologie évoquant parfois une organisation militaire. Parmi ses particularités, cette histoire comprend une fonction publique nombreuse, mais à l'effectif stable (51 145 agents permanents en 2016), répartie entre plusieurs centaines de métiers inscrits dans des filières technique, administrative, sociale et de santé, culturelle, chacune étant dotée d'une culture spécifique. La comparaison de la gestion municipale avec l'entretien d'un organisme vivant est fréquente.

Une analyse historique des 150 dernières années fait émerger l'évolution récente suivante : instaurée sous le Second Empire et prolongée sous la $\mathrm{III}^{\mathrm{e}}$ République, la "culture d'entreprise" Ville de Paris dont la figure de proue est alors le corps des ingénieurs agents de modernisation, et dont l'administration préfectorale s'est faite l'outil efficace, se voit transformée par des vagues successives d'évolutions intervenant dans des contextes socio-économiques divers, depuis la loi du 31 décembre 1975 rétablissant la fonction de Maire de Paris. Inscrire les cinquante dernières années dans une profondeur historique permettra de mettre en évidence tout à la fois ce souci de modernisation et d'innovation - conjugué à l'heure actuelle avec participation, aménité et propreté de la rue, verdissement et préoccupations environnementales - et certaines constantes ou retours, préoccupations mémorielles. Deux grandes périodes de restructuration se détachent plus particulièrement, dans les années 1980, puis depuis le début du XXI ${ }^{e}$ siècle, qui ont pour corollaire une perte d'identité professionnelle parmi les agents municipaux. 
Planche à semi (vers 1990), DEVE/École du Breuil. Outil vernaculaire au format de ces grandes barquettes de semis, utilisé pour y semer des graines de manière régulière.
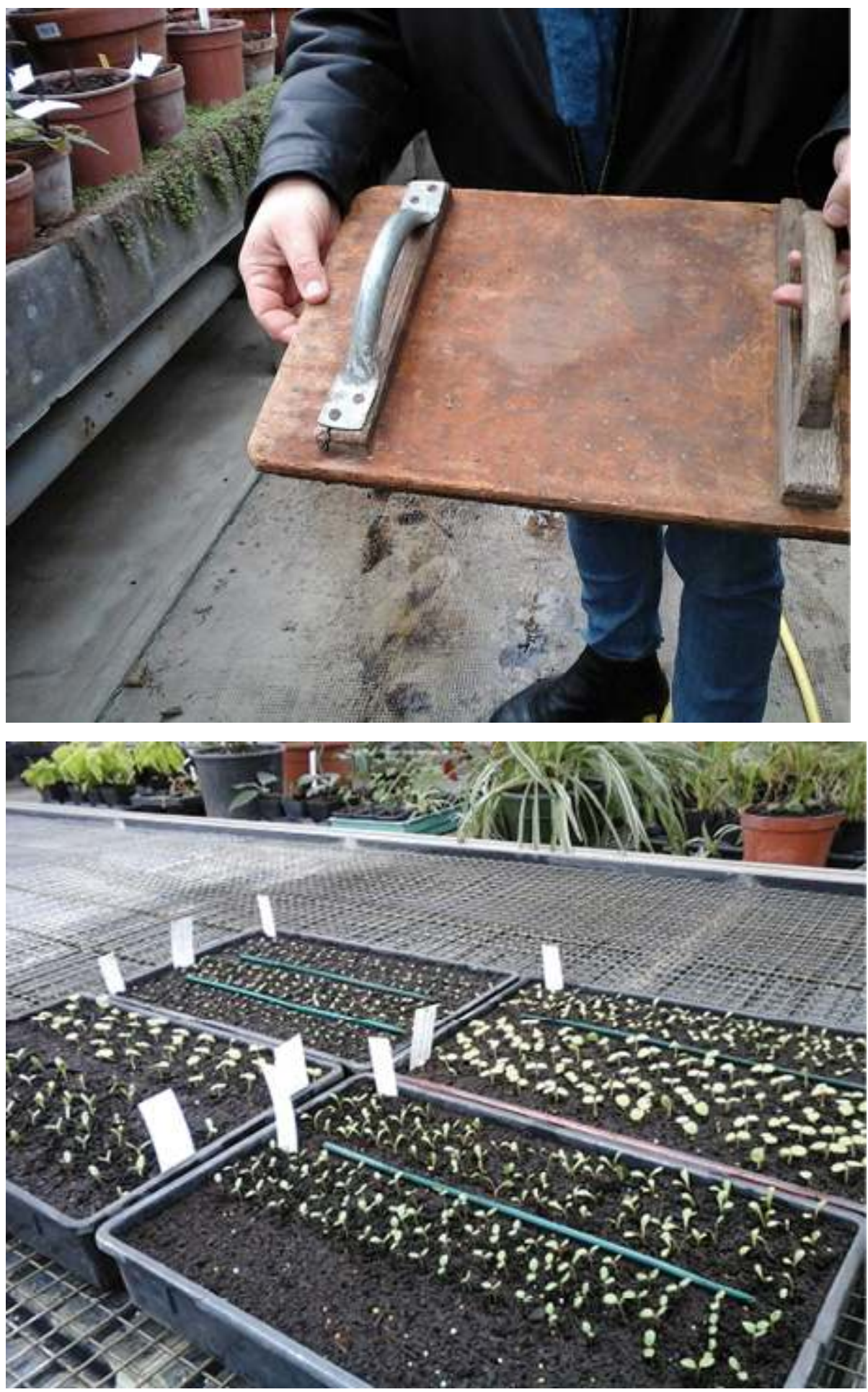

(C) Mairie de Paris/M. Simon

11 La question de l'échelle, a fortiori pour une organisation dont les activités sont aussi nombreuses et variées, est une donnée importante qui génère des lourdeurs, des temps de réaction longs et des cloisonnements. Révolution numérique, restructuration de l'administration, démocratie locale et importance plus grande accordée à la communication exercent une pression, tandis que des contraintes budgétaires accrues 
diminuent ou tarissent certains financements publics. Aussi est-il indispensable d'expliquer longuement la démarche, de rassurer, valoriser, convaincre, d'instaurer une relation de confiance qui nous engage personnellement et institutionnellement.

Historiquement, conservation va de paire avec destruction. Celle-ci peut ne laisser que des restes dus à la conjugaison de hasards, à nous de faire en sorte qu'elle devienne sélective, de trouver un juste équilibre entre patrimonialisation excessive et absence de patrimonialisation. Avant d'en être réduits à contempler la "beauté du mort", évitons aussi d'accélérer la mise à mort par une prédation pétrifiante. Les enjeux sont culturels, mais aussi sociaux et pourraient bien à terme devenir économiques : le patrimoine est vivant si l'on fait en sorte qu'il le reste. Cette réflexion guide constamment nos pas : cela passe par la conscience que nous devons opérer des choix avec discernement, cohérence et constance dans le temps. Cela passe également par une réhabilitation de la culture matérielle, du goût populaire pour les objets, le fait main, à la fois "relique" et objet de la curiosité du "comment ça marche" et des processus de transmission, dans une approche dynamique ${ }^{2}$. Cela passe enfin par la conscience que nous sommes collectivement des héritiers - de valeurs, droits, normes et procédures, mais aussi d'une histoire et d'une géographie qui donnent de l'épaisseur et de la substance à ces composantes - et des innovateurs. Nous devons notamment rétablir des ponts avec un passé très récent, la fin de l'administration préfectorale, en proposant des réponses en phase avec les aspirations qui s'expriment de façon de plus en plus lancinante (demande horizontale de participation, d'écoute et de "sur-mesure", multiplicité des approches et des réponses, souci d'économie et de bon usage des deniers publics), en nous inscrivant moins dans l'aménagement de dispositifs spectaculaires que dans la coordination de l'usage des richesses et dans une interaction respectueuse des savoirs de chacun, "petit patrimoine ordinaire" ou patrimoine de haute technologie (réseaux et laboratoires divers). Dans une ville où l'offre culturelle est immense, mais face à des mutations technologiques et économiques considérables, ce versant du patrimoine peut et doit permettre de développer de nouvelles formes d'attachement au local, de féconder les ressources d'une culture en constituant des collections qui seraient autant d'expressions à la fois d'une culture populaire, d'une "culture d'entreprise" et de leur patrimoine témoin, en recueillant des récits de métier et de carrière qui pourraient aussi prendre place dans le champ de l'accompagnement social.

Notre préoccupation essentielle est de faire en sorte que cette mission, pour l'instant calée sur la durée de la mandature municipale actuelle, soit utile et efficace. Pour cela, nous devons faire face à l'urgence des mutations ; donner un périmètre clair à notre intervention en la concentrant sur des objectifs réalistes ; permettre une appropriation active par les agents, en faisant en sorte qu'ils soient véritablement acteurs; penser dès l'amont à inscrire les objectifs dans la durée par différents moyens, et pour ce faire insuffler une culture patrimoniale dans l'organisation parisienne, sans quoi la mission ne serait qu'un fétu de paille éphémère produisant de la déception, voire du ressentiment. L'exercice est complexe. Nous devons agir dans la souplesse, avec une équipe réactive, agile, mais stable, bénéficiant d'autonomie et de confiance, sachant, dans le respect de chacun, rester à l'écoute des urgences et des demandes, tout en gardant le cap : sauvegarder la mémoire. 
Atelier école de pavage, DVD/CMA Centre de maintenance et d'approvisionnement

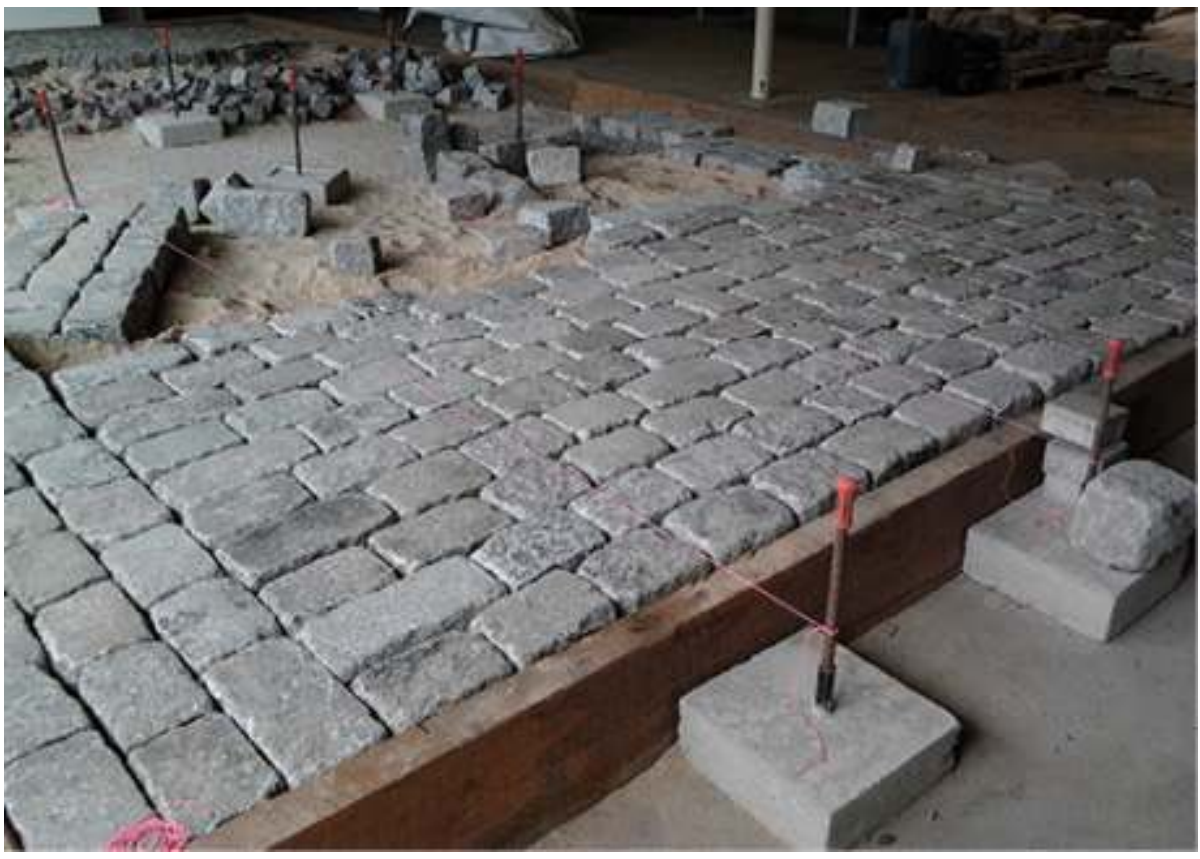

(c) Mairie de Paris/M. Simon

\section{Premier bilan d'activité}

Mettre en œuvre ce projet requérait la conjugaison de plusieurs compétences : trois personnes ont été recrutées en octobre 2016, par redéploiement au sein de la fonction publique parisienne, sur des fiches de poste en catégorie B de responsable de la documentation, administratif, multimédia, avec la perspective d'une polyvalence permettant une indispensable entraide mutuelle : ces personnes bénéficient d'une grande expérience au sein de l'organisation parisienne, deux d'entre elles sont des secrétaires administratifs chevronnés, la troisième est une photographe ayant expérimenté le multimédia dans ses fonctions antérieures. Certaines lacunes étaient à combler, qui ont requis une formation accélérée : techniques d'inventaire et de documentation, initiation à la constitution d'archives orales, conduite d'entretiens, filmage d'entretiens, de gestes et savoir-faire, de visites commentées, gestion des droits.

Dans tous les cas, il s'agissait d'inventer des méthodes adaptées à la commande, l'enjeu résidant dans le fait d'avoir à mener de front le recensement du patrimoine matériel et la constitution d'archives orales, qui doivent se nourrir mutuellement alors qu'habituellement, les deux sont dissociés ou que l'un est réduit à l'illustration de l'autre. Les critères d'unicité et de typicité président à la sélection des objets et des témoignages. Dans un premier temps, nous avons procédé à la mise au point de méthodologies que nous mettons à l'épreuve d'expérimentations de divers types, selon que nous les mettons en œuvre nous-mêmes (chantiers en interne), ou que nous les déléguons à des chercheurs extérieurs, en recueillant dans un premier temps l'avis de conseils scientifiques informels réunis sur deux chantiers. Nous prévoyons la mise en place d'un conseil scientifique permanent plus étoffé et statuant sur l'ensemble de nos 
activités à venir. Parallèlement, nous avons élaboré un plan de classement ainsi structuré :

- activité de la Mission Patrimoine Professionnel (notes, budget, fiches diverses : cession de droits, de recensement et d'inventaire ; expressions des besoins ; typologie et vocabulaire des métiers et des objets ; chronologie des arrêtés de structure et arborescence des organigrammes) ;

17 - bibliothèque et documentation générale ;

18 - collecte classée par direction, par service et par site : visites de repérage (fiche et dossier photographique), recensements et inventaires des objets d'usage et des objets fabriqués par les services (fiches assorties d'un dossier photographique et d'une documentation) ; audiovisuel (films et/ou enregistrements audio, rushes, fiches de synthèse, fiches chrono-thématiques).

19 Nous avons également entrepris la reconstitution des organigrammes, en nous appuyant sur divers annuaires administratifs et sur la chronologie des arrêtés de structure, tant il est indispensable de repérer finement les évolutions du squelette organisationnel de l'administration parisienne depuis quarante ans, soit sa municipalisation, indispensable pour situer les services dans leur profondeur temporelle. Bien que l'histoire administrative soit délaissée depuis deux décennies, ce travail constitue un préalable nécessaire pour permettre ultérieurement à des chercheurs de s'emparer de ce sujet majeur.

Les deux premiers chantiers menés par la Mission Patrimoine Professionnel (MPP), en raison de l'intérêt particulier de ces activités et sites et de leur déménagement imminent, portent sur le Laboratoire d'Essais des Matériaux, laboratoire appartenant à la Direction de la Voirie et des Déplacements (DVD) d'une part, et sur le Centre administratif d'autre part. Le premier, créé en 1868 afin d'éprouver la qualité des matériaux utilisés dans le cadre de la modernisation haussmannienne de Paris, était installé depuis lors dans l'un des pavillons Ledoux de la place Denfert-Rochereau et vient d'être transféré dans de nouveaux locaux mieux adaptés situés à la Porte de France ( $13^{\mathrm{e}}$ arrondissement), pour céder la place au musée de la Libération qui devrait y rouvrir ses portes fin 2019. Paradoxalement, ce laboratoire de physique et de chimie est internationalement réputé pour la qualité de ses analyses du ciment, mais méconnu au sein de la Ville de Paris, et ses agents déplorent un déficit de reconnaissance interne. Ce chantier a été mené en co-construction avec Sylvie Gosselin, ingénieure responsable de la prospective. Il a permis de recenser 132 objets et machines, et d'en inventorier 42 que la MPP transfère dans un local provisoire de conservation. Parallèlement, 14 entretiens - récits de métier et récits de carrière - ont été menés, 7 films de gestes et savoir-faire et une visite commentée filmée réalisés. Les premiers seront archivés et consultables sur autorisation, tandis que les autres films seront disponibles en ligne sur l'Intranet de la Ville, de même qu'un clip de communication. Une séance de restitution sera menée en décembre avec les agents interviewés, au cours de laquelle les films ayant vocation à être diffusés seront présentés et feront l'objet d'une discussion, à l'issue de laquelle leur sera remis le CD de leur interview. Ce chantier a été accompagné en début et fin, par un conseil scientifique qui a validé les méthodologies et salué la qualité de la démarche et des rendus, tandis que le directeur du laboratoire se dit très satisfait de son impact sur une équipe anxieuse de ce déménagement. 
Vue d'ensemble de la salle des essais au Laboratoire d'essais des matériaux LEM (place DenfertRochereau), DVD/LEM. Analyse après prélèvement en voirie de pastilles d'enrobé.

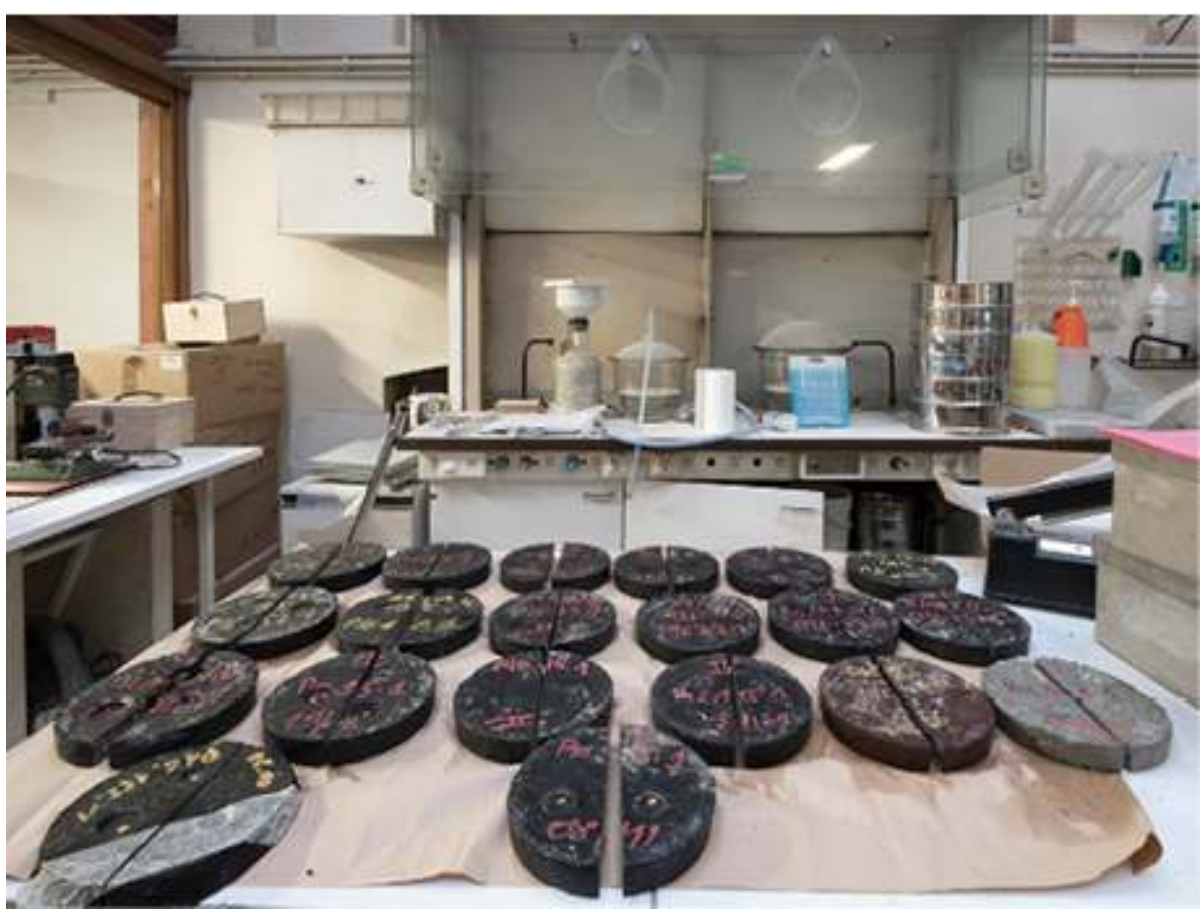

(c) Mairie de Paris/F. Morisson

Le Centre administratif situé 17, boulevard Morland ( $4^{\mathrm{e}}$ arrondissement), sera cédé en décembre dans le cadre de l'opération Réinventons Paris, tandis que les nombreux services hébergés depuis son inauguration en 1966, seront relogés sur divers sites parisiens dans un souci de regroupement de tous les services de chaque direction concernée. Conçu par le Prix de Rome Albert Laprade, cet édifice fit l'objet de louanges lors de son inauguration, tant pour les défis techniques à résoudre pour son édification (terrain alluvionnaire sur un ancien îlot de la Seine, passage en biais du métro en contrebas), que pour la modernité administrative dont il était porteur et que reflète la distribution intérieure : parking souterrain, premier restaurant en self-service, infirmerie et bibliothèque intégrées, permettant la journée continue ; importance accordée à l'accueil des administrés ; rationalisation des circulations intérieures (batteries d'ascenseurs, réseau pneumatique), mutualisation de certaines fonctionnalités. Ce chantier de recensement s'avère d'une grande complexité, en raison de la multiplicité des directions et services concernés. Le sauvetage d'objets et de mobilier de bureau intervient tardivement, mais demeure riche. Avec l'aide du responsable logistique du site, la MPP rencontre de nombreux acteurs présents ou retraités, et procède à des interviews qui requièrent un grand temps de préparation, tant les domaines d'activité y sont divers : Service du Plan de Paris, Logement et habitat, Finances et achats...

Parallèlement, un marché de constitution d'archives audiovisuelles porte sur les métiers ouvriers exercés au cimetière du Père-Lachaise, choisi pour la multiplicité de ses usages - site classé, à la fois cimetière, lieu de pèlerinage, lieu de promenade - et en raison de son relief qui interdit toute mécanisation pour son entretien. Les fossoyeurs, agents d'accueil des familles endeuillées, bûcherons-élagueurs, jardiniers et cantonniers, agents d'accueil et de surveillance, marquent tous une fierté et un 
attachement particulier au lieu, malgré des conditions physiques décrites comme particulièrement difficiles. L'enquête est menée par un sociologue du travail et une réalisatrice anthropologue, qui réalisent une vingtaine d'entretiens et plusieurs films de métier et remettront leurs livrables en fin d'année. Un conseil scientifique a été constitué. Par ailleurs, deux chercheuses procèdent à l'accompagnement d'éboueurs dans le but de constituer des archives orales sur le balayage manuel. Il est prévu également de procéder à la constitution d'archives orales des métiers de l'assainissement, dont des extraits seront présentés dans le cadre de la visite publique des égouts rénovée, qui devrait rouvrir ses portes à l'automne 2019.

Manutention d'un bateau-vanne au moyen d'un pont-roulant, DPE/STEA, Subdivision curage collecteurs et atelier d'entretien du matériel de curage des égouts

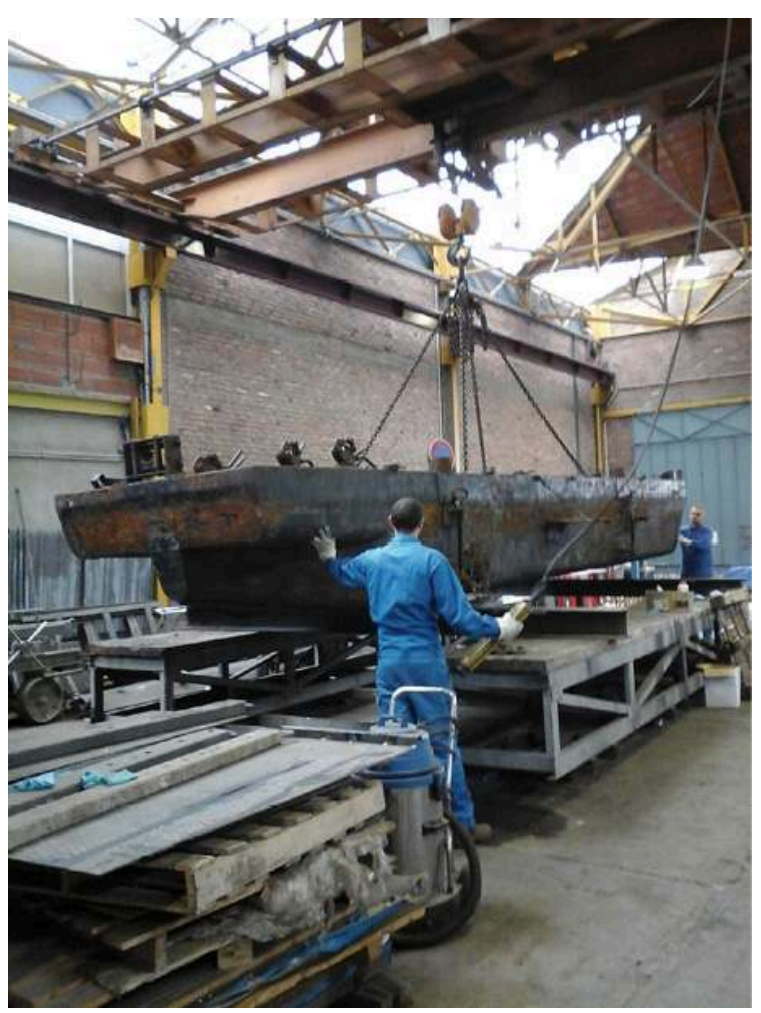

(c) Mairie de Paris/M. Simon

Enfin, la MPP demande aux archivistes en poste dans les diverses directions de leur signaler des agents riches d'une longue expérience au sein de la Ville de Paris et sur le point de prendre leur retraite, et procède elle-même à quelques interviews. Il ne s'agit pas de recherche, mais d'une démarche de recensement destinée à documenter l'histoire de l'organisation parisienne des quarante dernières années, et à ouvrir des portes à la recherche académique. Elle espère pouvoir intéresser celle-ci à l'intérêt de certains dossiers thématiques emblématiques des évolutions récentes dans leur approche municipale, comme par exemple le boulevard périphérique (construction, usages actuels, perspectives), ou l'habillement professionnel (porteur d'une identité professionnelle, évoluant dans le sens d'une meilleure protection "hygiène et sécurité" en concertation avec les syndicats). 


\section{Recentrage sur le patrimoine matériel}

La politique actuelle de regroupement de nombreux services, des budgets extrêmement compacts (baisse des dotations de l'État), enfin l'intense pression foncière exercée tant dans Paris intra-muros que par les municipalités de la banlieue proche où se situent nombre d'ateliers, garages et dépôts, amènent les décideurs à arbitrer en faveur d'un recentrage sur les déménagements imminents. L'approche par site reste pertinente en raison de l'inscription d'une activité dans un lieu et des bâtiments dont découle l'organisation physique des activités. Elle doit être croisée avec une approche par métier, par chaîne opératoire. Aussi procédons-nous à une priorisation des sites à recenser dans les trois années à venir, en fonction des dates prévues de leur transfert et du caractère particulier de leur activité. Parallèlement, nous élaborons un guide des bonnes pratiques à diffuser par le Secrétariat Général dans toutes les directions concernées, afin de sensibiliser la hiérarchie et les agents concernés sur la détermination de ce qui peut "faire patrimoine" et les aider à organiser le recensement et la conservation de ce patrimoine matériel, sous le contrôle de la MPP et avec l'aide de personnes ressources. Cela ne préjuge pas de l'abandon de la collecte de patrimoine immatériel, tant il est fondamental de contextualiser les objets et de les documenter.

Salle du pneumatique (1966), DILT/Cité administrative. Vestige du réseau pneumatique du courrier utilisé par l'administration de la Ville et du Département de Paris jusqu'en 2004.

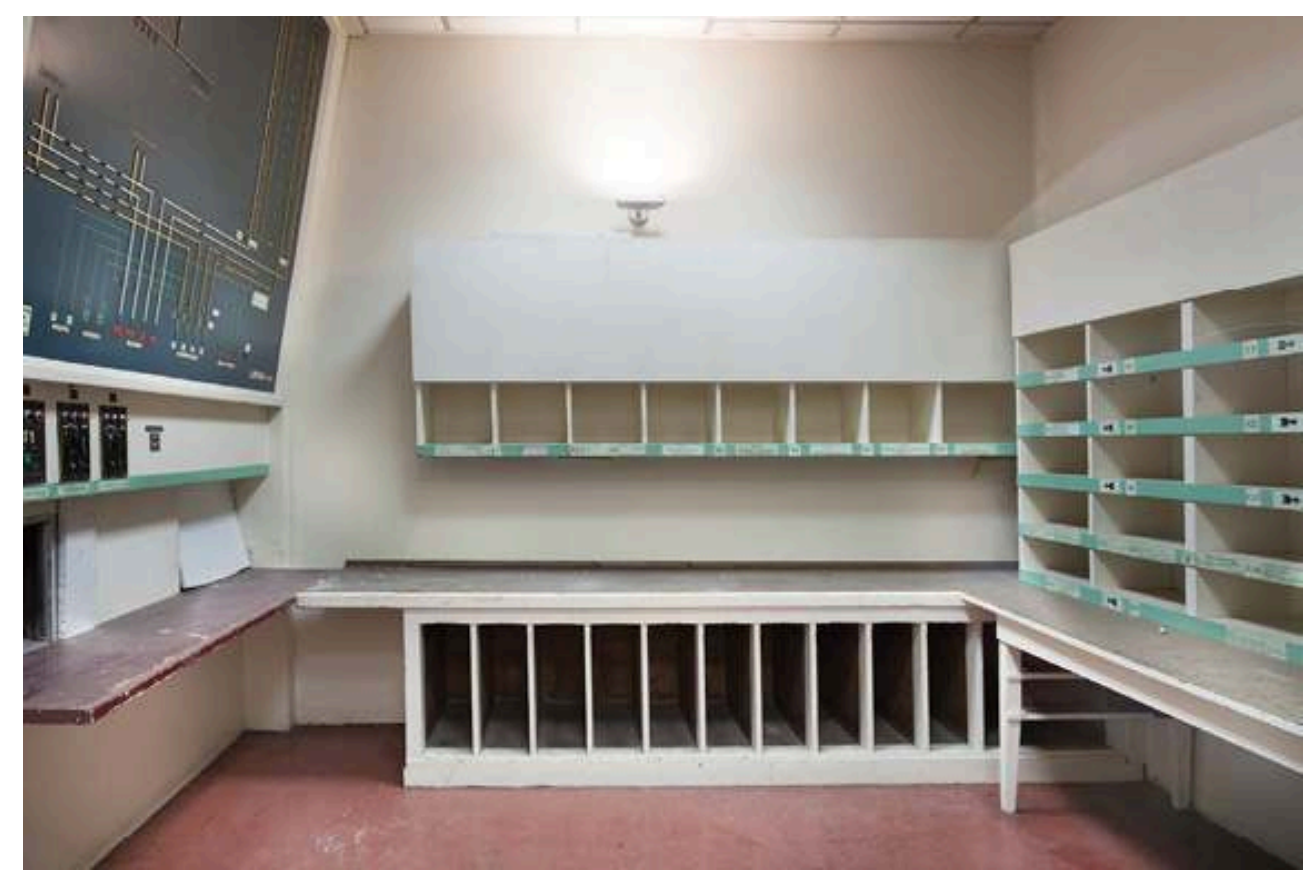

(C) Mairie de Paris/F. Morisson

Simultanément, nous réfléchissons à plusieurs options, complémentaires, de localisation physique de ce patrimoine, afin d'éviter ce qui serait perçu comme une prédation alors que dans bien des cas l'on observe un attachement aux objets et à leur valeur mémorielle et symbolique. Pour des raisons budgétaires, sociales et culturelles, nous préconisons donc des propositions multi-sites, avec la création ou le maintien d'espaces mémoriels déjà existants par des dispositifs muséographiques simples (vitrines sécurisées, facsimilés d'archives, signalétique pédagogique, veille par un 
récolement régulier) afin de favoriser une transmission des savoirs. Ces dispositifs se combineraient avec une réserve pérenne car, selon nous, le transfert forcément coûteux dans un local permanent doit être un dernier recours. Pour pallier les urgences, la MPP a obtenu le prêt très provisoire d'un local facile d'accès, mais de surface réduite ne permettant pas de stocker le matériel lourd et volumineux (mobilier, machines, poids lourds). L'aménagement d'un local pérenne est nécessaire pour les objets qui ne pourraient être conservés au sein des directions, et pour ceux dont la conservation est en péril. Dans tous les cas, la MPP devra anticiper les déménagements, les frais afférents, et accompagner ces déménagements.

Dans une perspective de valorisation qui ne préjuge pas d'autres moyens (expositions temporaires, organisation de visites lors des journées du patrimoine, création d'un centre de ressources...), à terme devraient être accessibles en ligne, à partir de 2020, avec la visée d'une interopérabilité des données avec d'autres bases, des films de savoirfaire, des visites commentées filmées, quelques films de communication, les fiches descriptives des objets inventoriés assorties de photographies et d'une documentation. Les interviews et rushes nettoyés, les fiches analytiques et chrono-thématiques des interviews, le fichier des objets recensés mais restant utilisés dans les services, seront consultables sur demande.

Nous avançons, avec un fort sentiment d'utilité et en faisant en sorte que notre action soit durable.

L'auteur adresse ses remerciements à Patrick Geoffray, directeur général de la DPE; Florence Descamps, École Pratique des Hautes Études; Marie-Noëlle Polino et Sylvère Aït-Amour, Rails et histoire ; Christiane Chanliau, Comité d'histoire du ministère de l'Environnement ; Isabelle Duhau et Jean Davoigneau, Bureau de la méthodologie de l'inventaire, ministère de la Culture ; Anne Nardin, musée Carnavalet.

Bortolotti, C. (dir.) Le patrimoine culturel immatériel : enjeux d'une nouvelle catégorie. Éditions de la Maison des sciences de l'Homme, Ethnologie de la France, n²6, 2011 Collectif, Sciences et techniques : une culture à partager, Culture et recherche, $\mathrm{n}^{\circ} 132$, automne-hiver 2015-2016.

Descamps, F. L'Historien, l'archiviste et le magnétophone : de la constitution de la source orale à son exploitation. Paris : Comité pour l'histoire économique et financière de la France, IGPDE, 2005.

DRH, Mission Analyses, Prévisions et Emplois, Commune de Paris et Département de Paris, Bilan social 2015, Mairie de Paris, 2016.

Drouguet, N. Le musée de société : de l'exposition de folklore aux enjeux contemporains. Paris : Armand Colin, 2015.

Heinich, N. La fabrique du patrimoine : de la cathédrale à la petite cuillère, Éditions de la Maison des sciences de l'Homme, Ethnologie de la France, n³1, 2009

Mollard, C. L'ingénierie culturelle et l'évaluation des politiques culturelles en France. Que sais-

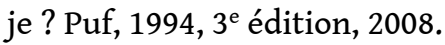

Pizzorni, F. Les musées de société : musées du XXI siècle : état des lieux et refondations.

Ministère de la Culture et de la Communication, Direction générale des patrimoines, Service des musées de France, juillet 2016.

Rails et Histoire : www.ahicf.com/ressources-documentaires.html Comité d'histoire du ministère de l'Environnement, de l'Énergie et de la Mer : www.developpement-durable.gouv.fr/-Le-Comite-d-histoire-du-ministere-.html 
PATSTEC, Mission nationale de sauvegarde du patrimoine scientifique et technique contemporain : www.patstec.fr/PSETT

RéMuT, Réseau national des musées techniques : http://remut.fr/

Ministère de la Culture : www.culture.gouv.fr/culture/inventai/patrimoine/;

www.culturecommunication.gouv.fr/Politiques-ministerielles/Inventaire-du-

patrimoine

\section{NOTES}

1. Nous assistons à une extension sans fin des périmètres typologique et temporel de ce qui fait patrimoine, au point que désormais pour certains, tout est patrimoine. Depuis la Révolution française, qui fit du monument un patrimoine, cette évolution étend le patrimoine jusqu'au paysage et à l'ordinaire, passant ainsi d'une acception restreinte de l'art à une définition extensive de la culture, au sens anthropologique de ce qui caractérise l'état d'une civilisation.

2. Pour ce faire, nous devons affronter une hiérarchie bien française qui veut que la culture matérielle est un parent pauvre méprisé, a fortiori celle du quotidien, et tenter d'en faire quelque chose qui puisse se partager afin de contribuer modestement à combler un hiatus aux racines anciennes, entre culture populaire et culture savante.

\section{RÉSUMÉS}

Depuis 2015, la Ville de Paris a entrepris un travail d'inventaire de son patrimoine professionnel matériel et immatériel : après une analyse du contexte, la responsable de cette mission présente la démarche suivie et la méthodologie adoptée ainsi que les premières actions qui préconisent une approche par lieux croisée à une approche par métiers et témoignent d'un souci d'insuffler une culture patrimoniale à l'organisation municipale.

\section{INDEX}

Mots-clés : conservation, patrimoine, patrimoine immatériel

\section{AUTEUR}

\section{MIRIAM SIMON}

conservatrice en chef du patrimoine, responsable de la Mission Patrimoine Professionnel, Mairie de Paris

miriam.simon@paris.fr 